\title{
Costo Promedio Ponderado de Capital para una mejor decisión de inversión
}

\author{
Weighted Average Cost of Capital for a better investment decision \\ Beatriz Sauza-Ávila ${ }^{a}$,José M. Cruz-Domínguez ${ }^{b}$ Suly S. Pérez-Castañeda ${ }^{c}$,Claudia B. \\ Lechuga-Canto ${ }^{d}$ Dorie Cruz-Ramírez ${ }^{e}$ Noé Chávez-Hernández ${ }^{f}$
}

\begin{abstract}
:
Every investor aims to maximize their assets in which they invest, which is why this research analyzes two large food business companies to find out the return they would offer to those who will reinvest their profits. This is achieved by calculating the Weighted Average Cost (CPPC) and when compared with the performance offered by companies in the same sector, in order for shareholders to make the decision that best suits them, the Yahoo Finance database was used to obtain the financial statements for the year 2020, from the economic units under study.
\end{abstract}

Keywords:

Financing, investment, decision.

Resumen:

Todo inversionista tiene como objetivo maximizar sus activos en los que invierte, es por ello que la presente investigación analiza dos grandes empresas de giro alimentario para conocer el rendimiento que ofrecería a quienes reinvertirán sus utilidades esto se logra al calcular el Costo Promedio Ponderado de Capital (CPPC) y al ser comparado con el rendimiento que ofrecen las empresas del mismo sector, para con ello los accionistas tomen la decisión que más les convenga, se hizo uso de la base de datos de Yahoo Finanzas para obtener los estados financieros del ejercicio 2020, de las unidades económicas en estudio.

\section{Palabras Clave:}

Financiamiento, inversión, decisión.

\section{Introducción}

Toda empresa independientemente de su tamaño necesita de financiamiento para llevar a cabo las operaciones ordinarias de la misma, este puede ser interno o externo, independientemente de la opción que se elija tiene implícito un costo, el cual pude llamarse costo de oportunidad cuando se da de manera interna y costo de financiamiento cuando se opta por recurrir a fuentes externas, ambos costos deben ser calculados, para con ello medir si las empresas pueden absorberlos y obtener rentabilidad, el objetivo de esta investigación es determinar el CPPC de dos empresas que servirá de base en la toma de decisiones en los inversionistas y comparar el porcentaje del rendimiento que puede tener el mismo sector.

\footnotetext{
Autor de Correspondencia, Universidad Autónoma del Estado de Hidalgo, https://orcid.org/0000-0002-7919-6792, Email: beatriz_sauza@uaeh.edu.mx

b Instituto Tecnológico Superior del Oriente del Estado de Hidalgo, https://orcid.org/0000-0003-2215-0694. Email: mcruz@ itesa.edu.mx

c Universidad Autónoma del Estado de Hidalgo, https://orcid.org/0000-0002-3763-9233, Email: sulysp@uaeh.edu.mx

${ }^{d}$ Universidad Autónoma del Estado de Hidalgo,https://orcid.org/0000-0003-3081-2379, Email: claublc @uaeh.edu.mx

e Universidad Autónoma del Estado de Hidalgo, https://orcid.org/0000-0002-7853-7655, Email: doriec@uaeh.edu.mx

f Tecnológico de Estudios Superiores de Coacalco, https://orcid.org/0000-0002-9165-2850 Email: nocahe@gmail.com
} 


\section{Desarrollo}

¿Qué es el costo promedio ponderado de capital CPPC? De acuerdo con Barajas (2013) es una medida financiera, la cual tiene el propósito de englobar en una sola cifra expresada en términos porcentuales, el costo de las diferentes fuentes de financiamiento que usará una empresa para fondear algún proyecto específico. De igual manera como asegura Escobar (2008), para los empresarios es de suma importancia conocer a detalle el costo de capital, considerando que para toda evaluación económica y financiera requiere tener una idea aproximada de ellos en las diferentes fuentes de obtener recursos que utiliza la empresa para emprender sus proyectos de inversión.

Toda empresa independientemente si es micro, pequeña, mediana o grande, requiere de contar con financiamiento que contribuya al desarrollo de sus actividades diarias, y con ello generar valor, de tal manera que sirva para obtener el mayor número de utilidades posibles, estas fuentes de financiamiento pueden ser internas o externas, las fuentes de financiamiento internas son las aportaciones que proporciona el dueño de la empresa o los socios y las externas pueden provenir de instituciones financieras, proveedores, o venta de acciones de la propia compañía. Como lo establece Intriago et al. (2021), el financiamiento es uno de los aspectos que permite a las empresas de cualquier tamaño o estructura tener recursos financieros para incrementar sus ingresos, adquirir 0 reemplazar activos, así como expandirse a corto, mediano y largo plazo.

\section{Fuentes de financiamiento}

Las fuentes de financiamiento se clasifican en dos internas y externas según Erossa (2020) describe a las internas de la siguiente manera:

a) Utilidades Reinvertidas: Esta fuente es muy común, los socios deciden que, en los primeros años, no repartirán dividendos, sino que estos son invertidos en la organización mediante la programación predeterminada de adquisiciones o construcciones (compras calendarizadas de mobiliario y equipo, según necesidades ya conocidas).

b) Aportaciones de los Socios: En el momento de constituir legalmente la sociedad (capital social) o mediante nuevas aportaciones con el fin de aumentar éste.

c) Emisión de acciones: Las acciones son títulos corporativos cuya principal función es atribuir al tenedor de la misma calidad de miembro de una corporación son títulos que se expiden de manera seriada y nominativa, esencialmente especulativas, quien adquiere una acción no sabe cuánto ganará, ya que se somete al resultado de los negocios que realice la Sociedad.

Las fuentes de financiamiento externar según López (2017) son:

a) Las aportaciones de los socios.

b) Préstamos, ejemplos de ello puede ser a través del Sistema Financiero Mexicano como son los bancos, los cuales cobrarán intereses y puede solicitarse a corto o largo plazo.

c) Leasing, que es un contrato por el cual una empresa concede a otra el uso de un bien a cambio del pago de unas cuotas de alquiler periódicas durante un determinado periodo de tiempo. Al terminar el contrato, el usuario del bien o arrendatario dispondrá de una opción de compra sobre el bien. Es una fuente de financiamiento a largo plazo.

d) Factoring, que consiste en que una sociedad cede a otra el cobro de sus deudas.

e) Confirming, que es un producto financiero en donde una empresa (cliente) entrega la administración integral de los pagos a sus proveedores (beneficiarios) a una entidad financiera o de crédito.

f) Pagaré, que es un documento que supone la promesa de pago a alguien. Este compromiso incluye la suma fijada de dinero como pago y el plazo de tiempo para realizar el mismo.

g) Crowdfounding, también llamado micromecenazgo, que consiste en financiar un proyecto a través de donaciones colectivas.

h) Crowdlending, que es un mecanismo de financiación colectiva por el que pequeños inversores prestan su dinero a una empresa con el objetivo de recuperar la inversión en un futuro sumada al pago de unos intereses.

i) Venture Capital, que son inversiones a través de acciones que sirven para financiar compañías de pequeño o mediano tamaño.

Una fuente de financiamiento externa más común en las empresas y que no se menciona en el anterior listado son los proveedores, a quienes se les compra mercancía a crédito como lo menciona García et al. (2004) al decir que las empresas negocian su financiamiento con los proveedores. 


\section{Metodología}

Pasos para determinar el CPPC de acuerdo con el modelo propuesto del Colegio de Contadores Públicos de México (CCPM).

1. Se calcula el porcentaje de participación que tiene cada componente sobre el total de la estructura de financiamiento.

2. Se considera la deuda a corto y largo plazo que se haya obtenido la cual tiene un costo antes de impuestos. Se investiga el costo de las acciones preferentes, acciones ordinarias, utilidades retenidas, y también se considera el Impuesto Sobre la Renta vigente

3. Debe determinarse el promedio ponderado de los diferentes costos de las fuentes de financiamiento. Este ponderado se calcula multiplicando el porcentaje de participación (\% relativo) de cada componente del financiamiento por su correspondiente costo financiero. Después se suman estos resultados. La sumatoria es el costo promedio ponderado de capital.

4. Se determina la tasa de rentabilidad para conocer si será mayor al CPPC para poder ser atractiva para quienes decidan invertir.

Para poder aplicar la metodología del CPPC del CCPM, se obtuvieron los estados financieros de dos empresas de giro alimentario de la base de datos de Yahoo Finanzas, por ser una plataforma de acceso libre.

\section{Resultados}

A continuación, se presenta el análisis de las dos empresas alimentarias utilizando la metodología del CPPC, así como el cálculo de la rentabilidad.

\section{Empresa giro alimentario 1}

La empresa uno ha obtenido en el ejercicio 2021 un financiamiento externo por $\$ 219,640,000.00$, sus fuentes de financiamiento son acciones ordinarias por un monto de $\$ 4,061,000.00$, utilidades retenidas por $\$ 64,265,000.00$ obteniéndose un total de inversión financiada tanto interno como externo de $\$ 287,966.000 .00$, como se muestra en la Tabla 1.

Tabla 1. Total de patrimonio

\begin{tabular}{lll}
\multicolumn{1}{c}{ Concepto } & \multicolumn{1}{c}{ Monto } \\
\hline Deuda a corto y largo plazo & $\$$ & $219,640,000.00$ \\
\hline Acciones ordinarias & $4,061,000.00$ \\
\hline $\begin{array}{l}\text { Utilidades retenidas } \\
\text { Total, pasivo y } \\
\text { patrimonio }\end{array}$ & $64,265,000.00$ \\
\hline
\end{tabular}

Fuente: Elaboración propia con estados financieros de Yahoo Finanzas (2021)
Después de obtener la información que arroja en el estado financiero de la empresa giro alimentario 1 se procede al calcular los valores absolutos y relativos quedando como se muestra en la Tabla 2, se observa que el $76.27 \%$ está integrado por deuda externa, el $1.41 \%$ está representado por acciones ordinarias, el $22.32 \%$ corresponde a utilidades retenidas.

Tabla 2. Valor absoluto y relativo

\begin{tabular}{llrr}
\multicolumn{1}{c}{ Fuente } & \multicolumn{1}{c}{ Cálculo } & Absoluto & Relativo \\
\hline $\begin{array}{l}\text { Deuda a corto y } \\
\text { largo plazo }\end{array}$ & $\begin{array}{c}219640000 / \\
287966000\end{array}$ & 0.762728933 & $76.27 \%$ \\
\hline Acciones ordinarias & $4061000 / 287966000$ & 0.014102359 & $1.41 \%$ \\
\hline $\begin{array}{l}\text { Utilidades retenidas } \\
\text { Total, pasivo y }\end{array}$ & $64265000 / 287966000$ & 0.223168707 & $22.32 \%$ \\
patrimonio & $\mathbf{2 8 7 , 9 6 6 , 0 0 0 . 0 0}$ & $\mathbf{1}$ & $\mathbf{1 0 0 \%}$
\end{tabular}

Fuente: Elaboración propia con la metodología de CCPM

De acuerdo con lo que propone el CCPM (2014), todo financiamiento ya sea interno o externo tiene un costo, por ello en la Tabla 3 se muestra en la columna de nombre costo (z), el costo que representa la deuda a corto y largo plazo es del $27 \%$, considerando que la empresa paga el $30 \%$ del Impuesto Sobre la Renta, por lo tanto los intereses que se generan por este financiamiento son deducibles, se tiene un costo de deuda a corto y largo plazo de $18.90 \%$, el costo aproximado de las acciones ordinarias es del $3.50 \%$, y el costo aproximado de las utilidades retenidas es de $3.68 \%$. Posteriormente para determinar el cálculo de la columna $\left(Y^{\star} Z\right)$, se multiplica el valor relativo por el costo con ello se obtiene el promedio ponderado de los costos, la suma de esta columna representa el CCPP, el cual es equivalente al $15.29 \%$.

Tabla 3. Determinación del CPPC

\begin{tabular}{|c|c|c|c|}
\hline Fuente & $\begin{array}{c}\text { \% Relativo } \\
(\mathrm{Y})\end{array}$ & $\begin{array}{c}\text { Costo } \\
\text { (Z) }\end{array}$ & $(Y * Z)$ \\
\hline $\begin{array}{l}\text { Deuda a corto y } \\
\text { largo plazo }\end{array}$ & $\%$ & 18. & $14.42 \%$ \\
\hline $\begin{array}{l}\text { Acciones } \\
\text { preferentes }\end{array}$ & $0.00 \%$ & $0.00 \%$ & $0.00 \%$ \\
\hline Acciones ordinarias & $1.41 \%$ & $3.50 \%$ & $0.05 \%$ \\
\hline Utilidades retenidas & $22.32 \%$ & $3.68 \%$ & $0.82 \%$ \\
\hline $\begin{array}{l}\text { Total, promedio } \\
\text { ponderado } \\
\text { Fuente: Elabora }\end{array}$ & $100.00 \%$ & & $15.29 \%$ \\
\hline
\end{tabular}

A continuación, se determina el retorno esperado el cual es de $\$ 44,019,952.00$, como se muestra en la tabla 4 . 
Tabla 4. Retorno esperado

\begin{tabular}{lcc}
\multicolumn{1}{c}{ Concepto } & Desarrollo & Total \\
\hline Intereses deuda & $219640000 \times 27 \%$ & $\$ 59,302,800.00$ \\
\hline Menos ahorro de impuestos & & $17,790,840.00$ \\
\hline Costos Intereses netos (1) & & $41,511,960.00$ \\
\hline $\begin{array}{l}\text { Retorno esperado Accionistas } \\
\text { Ordinarios (3) }\end{array}$ & $4061000 \times 2.50 \%$ & $142,135.00$ \\
\hline $\begin{array}{l}\text { Retorno esperado Utilidades } \\
\text { Retenidas (4) }\end{array}$ & $64265000 \times 2.68 \%$ & $2,364,952.00$ \\
\hline $\begin{array}{l}\text { Retorno esperado (1+2+3+4) } \\
\text { Fuente: Elaboración }\end{array}$ & $\begin{array}{c}\text { 287966000 X 15.29\% } \\
\text { propia con la metodología de } \\
\text { CCPM }\end{array}$ & $\begin{array}{c}\$ 44,019,047.00 \\
\end{array}$
\end{tabular}

De acuerdo con lo sugerido el CCPM (2014), se puede comprobar el resultado por el modelo matemático siguiente:

$$
\mathbf{C P P C}=(\mathrm{Wd} \times \mathrm{Tdi})+(\mathrm{Wac} \times \mathrm{Kac})+(\mathrm{Wr} \times \mathrm{Kr})
$$

Donde:

Wd= Participación de la deuda en la Estructura $\mathrm{Tdi}=$ Tasa de interés de la deuda después de impuestos Wac $=$ Participación de las acciones ordinarias $\mathrm{Kac}=$ Costo de capital de las acciones ordinarias $\mathrm{Wr}=$ Participación de las utilidades retenidas $\mathrm{Kr}=$ Costo de las utilidades retenidas

Sustituyendo los valores se obtiene un valor de CPPC= $44,019,047.00$, para finalizar se determina la tasa de rendimiento esperado con la inversión de las fuentes de inversión interna y externas utilizando la siguiente fórmula:

$$
\operatorname{Tr}=\mathrm{CPPC} / \mathrm{TF}
$$

$\mathrm{Tr}=\mathrm{Tasa}$ de rendimiento esperado

$\mathrm{CPPC}=$ Costo promedio ponderado de capital

$\mathrm{TF}=$ Total financiero

Sustituyendo los valores se obtiene el resultado de $\mathrm{Tr}$ como se muestra en la Tabla 5.

Tabla 5 Tasa de rendimiento esperado

\begin{tabular}{lrr}
\multicolumn{2}{c}{ Concepto } & \multicolumn{2}{c}{ Monto } \\
\hline $\operatorname{Tr}$ & \multicolumn{2}{c}{$\$$} \\
\hline CPPP & $44,019,047.00$ \\
\hline Total Financiamiento & $\$$ & $287,966,000.00$ \\
\hline $\operatorname{Tr}$ & & 0.152861959 \\
\hline $\operatorname{Tr}$ & $\mathbf{1 5 . 2 9 \%}$ \\
Fuente: Elaboración propia con la metodología de \\
\multicolumn{3}{c}{ CCPM }
\end{tabular}

Se deduce que con el modelo matemático y con el promedio ponderado de los diferentes costos de las fuentes de financiamiento multiplicado por el porcentaje de participación relativo al sumar estos valores, en ambos casos se llega al mismo porcentaje de CPPC de $15.29 \%$. esto significa que la empresa debe obtener un rendimiento mayor a este porcentaje para que sea atractiva la reinversión de utilidades en los accionistas.

\section{Empresa giro alimentario 2}

La empresa uno ha obtenido en el ejercicio un financiamiento externo por $\$ 46,104,385.00$, sus fuentes de financiamiento externo son acciones ordinarias por un monto de $\$ 4,924,197.00$ utilidades retenidas por $\$ 20,596,419.00$ obteniéndose un total de inversión financiada tanto interno como externo de $\$ 71,625,001.00$, como se muestra en la Tabla 6.

\begin{tabular}{|c|c|}
\hline Concepto & Total \\
\hline Deuda a corto y largo plazo & $46,104,385.00$ \\
\hline Acciones preferentes & . \\
\hline Acciones ordinarias & $4,924,197.00$ \\
\hline Utilidades retenidas & $20,596,419.00$ \\
\hline $\begin{array}{r}\text { Total pasivo y patrimonio } \\
\text { ente: Elaboración prop } \\
\text { Yahoo Fir }\end{array}$ & $\begin{array}{l}\$ \quad \mathbf{7 1 , 6 2 5 , 0 0 1 . 0 0} \\
\text { con estados financie } \\
\text { nzas (2021) }\end{array}$ \\
\hline
\end{tabular}

Tabla 6 Total patrimonio

Después de obtener la información que arroja en el estado financiero de la empresa giro alimentario 2 se procede al calcular los valores absolutos y relativos quedando como se muestra en la Tabla 7 , se observa que el $64.37 \%$ esta integrado por deuda externa, el $6.87 \%$ está representado por acciones ordinarias, el $28.76 \%$ corresponde a utilidades retenidas.

\begin{tabular}{|c|c|c|c|}
\hline Fuente & Cálculo & Absoluto & Relativo \\
\hline $\begin{array}{l}\text { Deuda a corto y } \\
\text { largo plazo }\end{array}$ & $46104385 / 71625001$ & 0.64369123 & $64.37 \%$ \\
\hline $\begin{array}{l}\text { Acciones } \\
\text { ordinarias }\end{array}$ & $4924197 / 71625001$ & 0.068749695 & $6.87 \%$ \\
\hline $\begin{array}{l}\text { Utilidades } \\
\text { retenidas }\end{array}$ & $20596419 / 71625001$ & 0.287559075 & $28.76 \%$ \\
\hline $\begin{array}{l}\text { Total pasivo y } \\
\text { patrimonio } \\
\text { Fuente: Ela }\end{array}$ & $\begin{array}{r}71,625,001.00 \\
\text { coración propia } \\
\text { CCPM }\end{array}$ & 1 & $\begin{array}{r}100 \% \\
\text { gía de }\end{array}$ \\
\hline
\end{tabular}

Tabla 7. Valor absoluto y relativo

De acuerdo con lo que propone el CCPM (2014), todo financiamiento ya sea interno o externo tiene un costo, por ello en la tabla 8 se muestra en la columna de nombre costo (z), el costo que representa la deuda a corto y largo plazo es del $27 \%$, considerando que la empresa para el $30 \%$ del Impuesto Sobre la Renta, por lo tanto los intereses que se generan por este financiamiento son deducibles considerando esta deducción se tiene un costo de deuda a corto y largo plazo de $18.90 \%$, el costo aproximado de las acciones ordinarias es del $3.50 \%$, y el costo aproximado de las utilidades retenidas es de $3.68 \%$. Posteriormente para determinar el cálculo de la columna 
$\left(Y^{\star} \mathrm{Z}\right)$, se multiplica el valor relativo por el costo con ello se obtiene el promedio ponderado de los costos, la suma de esta columna representa el CCPP, el cual es equivalente al $13.46 \%$ (Tabla 8 ).

Tabla 8. Determinación del CPPC

\begin{tabular}{|c|c|c|c|}
\hline Fuente & $\begin{array}{c}\% \text { Relativo } \\
(Y)\end{array}$ & Costo (Z) & $\left(Y^{*} \mathbf{Z}\right)$ \\
\hline $\begin{array}{l}\text { Deuda a corto y } \\
\text { largo plazo }\end{array}$ & $64.37 \%$ & $18.90 \%$ & $12.17 \%$ \\
\hline Acciones ordinarias & $6.87 \%$ & $3.50 \%$ & $0.24 \%$ \\
\hline Utilidades retenidas & $28.76 \%$ & $3.68 \%$ & $1.06 \%$ \\
\hline $\begin{array}{l}\text { Total promedio } \\
\text { ponderado } \\
\text { Fuente: Elaborac }\end{array}$ & $\begin{array}{c}100.00 \% \\
\text { propia co } \\
\text { CCPM }\end{array}$ & 0.007 & $\begin{array}{l}13.46 \% \\
\text { logía de }\end{array}$ \\
\hline
\end{tabular}

A continuación, se determina el retorno esperado el cual es de $\$ 9,644,023.88$, como se muestra en la Tabla 9.

Tabla 9. Retorno esperado

\begin{tabular}{|c|c|c|}
\hline Concepto & Despeje & Total \\
\hline Intereses deuda & $46,104,385.00 \times 27 \%$ & $12,448,183.95$ \\
\hline Menos ahorro de impuestos & & $3,734,455.19$ \\
\hline Costos Intereses netos (1) & & $8,713,728.77$ \\
\hline $\begin{array}{l}\text { Retorno esperado Accionistas } \\
\text { Ordinarios (2) }\end{array}$ & $4,924,197.00 \times 2.50 \%$ & $172,346.90$ \\
\hline $\begin{array}{l}\text { Retorno esperado Utilidades } \\
\text { Retenidas ( } 3 \text { ) }\end{array}$ & $20,596,419.00 \times 2.68 \%$ & $757,948.22$ \\
\hline $\begin{array}{l}\text { Retorno esperado }(1+2+3) \\
\text { Fuente: Elabora }\end{array}$ & $\begin{array}{l}71,625,001.00 \times 13.46 \% \\
\text { propia con la met }\end{array}$ & $\begin{array}{l}9,644,023.88 \\
\text { ogía de }\end{array}$ \\
\hline
\end{tabular}
CCPM

De acuerdo con lo sugerido el CCPM (2014), se puede comprobar el resultado por el modelo matemático siguiente:

$$
\mathbf{C P P C}=(\mathrm{Wd} \times \mathrm{Tdi})+(\mathrm{Wac} \times \mathrm{Kac})+(\mathrm{Wr} \times \mathrm{Kr})
$$

Sustituyendo los valores se obtiene un valor de CPPC= $9,644,023.88$ para finalizar se determina la tasa de rendimiento esperado con la inversión de las fuentes de inversión interna y externas utilizando la siguiente fórmula:

$\mathrm{Tr}=\quad$ Tasa de rendimiento esperado

$\mathrm{CPPC}=$ Costo promedio ponderado de capital

$\mathrm{TF}=$ Total financiero

Sustituyendo los valores se obtiene el resultado de $\mathrm{Tr}$ como se muestra en la Tabla 10.

\section{$\mathrm{Tr}=\mathrm{CPPP} / \mathrm{TF}$}

$\mathrm{Tr}=$ Tasa de rendimiento esperado

CPPP= Costo promedio ponderado de capital

TF= Total Financiamiento
Tabla 10 Tasa de rendimiento esperado

\begin{tabular}{cr} 
Concepto & \multicolumn{1}{c}{ Total } \\
\hline CPPP & $9,644,023.88$ \\
\hline Total Financiamiento & $71,625,001.00$ \\
\hline $\operatorname{Tr}$ & 0.134646056 \\
\hline Fr & $\mathbf{1 3 . 4 6 \%}$ \\
Fuente: Elaboración propia con la metodología de \\
CCPM
\end{tabular}

Se deduce que con el modelo matemático y con el promedio ponderado de los diferentes costos de las fuentes de financiamiento multiplicado por el porcentaje de participación relativo al sumar estos valores, en ambos casos se llega al mismo porcentaje de CPPC de $13.46 \%$. esto significa que la empresa debe obtener un rendimiento mayor a este porcentaje para que sea atractiva la reinversión de utilidades en los accionistas.

De acuerdo con la revista digital de Expansión (2012) una empresa de giro alimentario tiene un rendimiento del $15.3 \%$, al compararse el CPPC obtenido en cada una de las empresas de estudio para que estas sean competitivas deben ser mayores a los resultados obtenidos a los que produce el mismo sector. En este caso la única empresa que se encuentra con un porcentaje menor del CPPC es la empresa 2 que es del $13.46 \%$ la diferencia representa un $1.84 \%$, en este caso los accionistas pueden optar por elegir en no reinvertir sus utilidades.

\section{Conclusión}

De acuerdo con la revista digital expansión el rendimiento de una empresa alimentaria es del $15.3 \%$, para la empresa giro alimentario 1 con un valor de CPPC del $15.29 \%$ y para la empresa giro alimentario 2 con valor de CPPC del $13.46 \%$, los accionistas de la empresa 1 pueden seguir optando por seguir reinvirtiendo sus utilidades ya que el rendimiento es casi igual al rendimiento de empresas del mismo sector, sin embargo para la empresa 2 los accionistas pueden decidir en no reinvertir sus utilidades y tener la opción de realizar inversiones en otra empresa por existir una diferencia del $1.84 \%$ de rendimientos de acuerdo a lo reportado por empresas del mismo sector.

Al aplicar la metodología sugerida por el CCPM utilizando el CPPC, se concluye que las grandes empresas pueden llevar un control constante del rendimiento que puede tener su empresa al hacer uso de este modelo y con ello servirá para que los accionistas puedan tomar decisiones que les permitan maximizar sus utilidades. 


\section{Referencias}

CCPM. (2014). Finanzas y Sistema Financiero. Boletín de Investigación de la Comisión de Desarrollo Finanzas y Sistema Financiero, 16, pp. 114.

Barajas, S. (2013). ¿Qué uso tiene y como se calcula el Costo Promedio Ponderado de Capital?. Forbes 14. Recuperado el 23 de enero de 2021, de https://www.forbes.com.mx/que-uso-tiene-y-como-secalcula-el-costo-de-capital-promedio-

ponderado/\#: :text=El\%20Costo $\% 20 \mathrm{de} \% 20 \mathrm{Capital} \% 20$ Promedio $\%$ 20Ponderado\%20(CCPP)\%20es\%20una\%20medida,fondear\%20alg $\%$ C3\%BAn\%20proyecto\%20en\%20espec\%C3\%ADfico.

Escobar, G.E. (2008). Las empresas de alimentos de Manizales generan ganancia, pero destruye valor económico agregado (EVA) ANFORA, Vol. 15(), pp, 317-336. Recuperado el 28 de enero de 2021, de https://www.redalyc.org/articulo.oa?id=357834257015.

Erossa, V.E. (2020). Fuentes de Financiamiento a Mediano y Largo Plazo, y su Costo, apuntes de la asignatura de Proyectos de Inversión en Ingeniería, recuperado el 27 de enero de 2021, de https://www.fcca.umich.mx/descargas/apuntes/academia\%20de $\% 20$ finanzas/finanzas $\% 20 \mathrm{ii} \% 20$ mauricio $\% 20 \mathrm{a} . \% 20$ chagolla $\% 20$ farias/a dministracion\%20financiera\%20capitulo\%206.pdf

Expansión (2012). Alimentos y bebidas, inversión exitosa. Recuperado el 20 de enero de 2021, de https://expansion.mx/midinero/2012/06/20/apuesta-tu-inversion-a-las-marcas.

García, C.A., Ochoa, N., Zaragoza G. y Pech, J. (2004). Desarrollo de una herramienta computacional para el análisis de financiamiento de las obras que incluya las obligaciones tributarias y las condiciones de pago a proveedores, 8(2), pp. 91-102.

Intriago, M.E., Bravo, N.G. y Bravo, D.C. (2021). Una aproximación preliminar a la gestión de los recursos financieros de las pequeñas y medianas empresas del sector agrícola. Vol. 15 (1), pp. 1-10.

López, D. (2017) Fuente de financiamiento. Economipedia.com, recuperado el 21 de enero de 2021 en https://economipedia.com/definiciones/fuente-de-financiacion.html

Yahoo Finanzas (2021). Estados financieros, recuperado el 21 de enero de 2021, de https://es-us.finanzas.yahoo.com 